\title{
EFEITOS BIOLÓGICOS DAS RADIAÇÕES NÃO-IONIZANTES : NORMAS DE SEGURANÇA PARA A EXPOSIÇÃO A CAMPOS DE RÁDIO-FREQÜÊNCIAS E MICROONDAS.
}

\author{
José Thomaz Senise \\ Instituto Mauá de Tecnologia
}

\begin{abstract}
Sumário: As normas de segurança para exposição a campos eletromagnéticos baseiam-se em critérios que foram sendo aperfeiçoados com o tempo. O principal objetivo deste artigo é mostrar essa evolução e expor alguns conceitos básicos, de forma a facilitar o exame crítico do grande volume de publicações sobre o assunto e esclarecer opiniões freqüentemente contraditórias.
\end{abstract}

\begin{abstract}
This paper's main objective is to show how safety standards for human exposure to electromagnetic fields have evolved with time. Basic concepts are emphasized, thus permitting a critical reading of the vast literature on the subject and a better judgment on often confusing opinions.
\end{abstract}

Palavras-Chave: Efeitos biológicos; Normas de Segurança; Radiações não-ionizantes; Exposição a RF e microondas.

\section{INTRODUÇÃO}

Os efeitos biológicos dos fenômenos elétricos são conhecidos há muito tempo. Certamente há mais tempo do que os primeiros registros de choques elétricos provocados por descargas de garrafas de Leyden, no século 18 (1745) [1].

A partir da invenção da pilha de Volta, em 1800 , desenvolveram-se inúmeras aplicações médicas de corrente contínua. D'Arsonval (n.1851), provavelmente, foi o primeiro a estudar os efeitos fisiológicos da corrente alternada e de campos elétricos e magnéticos variáveis.

No início do século 20 , os efeitos térmicos dos campos de rádio-freqüência eram bem conhecidos e utilizados em diatermia.

Ao longo da história, a introdução de novos aparelhos e novas técnicas relacionadas com a eletricidade provocou e continua a provocar reações pessoais e coletivas de todo tipo.
Há 150 anos, em 1846, quando Morse iniciava a instalação das primeiras linhas telegráficas, um jornal de Filadélfia publicava artigo relacionando o aumento de chuvas locais à presença daquelas linhas [2].

Avisos afixados em quartos de hotéis, 50 anos mais tarde, procuravam desfazer a crença em efeitos nocivos à saúde dos hóspedes, inclusive a insônia, atribuidos às lâmpadas elétricas recentemente instaladas .

Hoje em dia, após muitos anos de uso generalizado dos fornos de microondas domésticos, ainda há quem não confie nos mesmos, relacionando microondas com radioatividade, acreditando na toxicidade de alimentos cozinhados nesses fornos e em outras tantas inverdades que compõem o folclore criado em volta dessa peça quase indispensável numa cozinha moderna.

Somente um conhecimento melhor das normas de segurança, alicerçadas em décadas de trabalhos científicos, pode levar as pessoas a um exame crítico das dúvidas levantadas em relação aos efeitos de uma exposição à radiaçāo eletromagnética.

\section{CONCEITOS BÁSICOS}

Em primeiro lugar, lembramos que a palavra "radiação", comumente associada a radioatividade, raios $\mathrm{X}$ e outros "perigos", refere-se, de maneira geral, a "energia radiante" : energia que se propaga no espaço, inclusive no vácuo, sem necessidade de um suporte físico. Em particular, "radiação eletromagnética" é energia que se propaga sob a forma de ondas caracterizadas por campos elétricos e magnéticos variáveis no tempo e no espaço.

As ondas eletromagnéticas podem ser identificadas por três parâmetros interdependentes (v.fig.1): a frequiência $\mathbf{f}$ $(\mathrm{Hz})$, o comprimento de onda $\lambda(\mathrm{m})$ e a energia do fóton E $(\mathrm{J}$ ou eV), entre os quais valem as relações : $f=v / \lambda e$ $\mathrm{E}=\mathrm{hf}$, onde $\mathbf{v}$ é a velocidade de propagação da onda (no

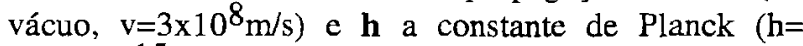
$4,14 \times 10^{-15} \mathrm{eV} / \mathrm{Hz}$ ). 


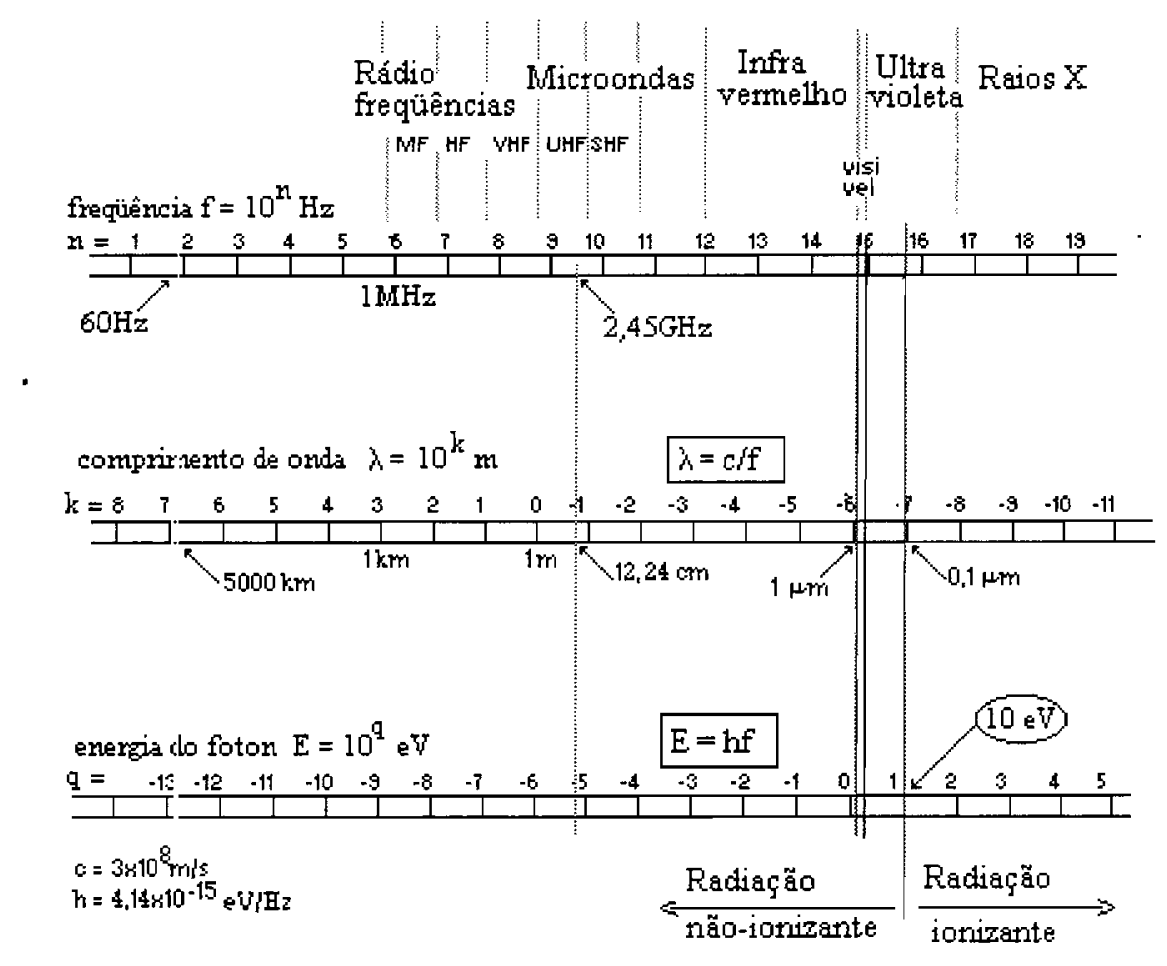

\section{fig.1}

Consideram-se radiações ñão-ionizantes todas as radiações eletromagnéticas com energia do fóton inferior a $10 \mathrm{eV}$, valor esse mínimo para "arrancar" elétrons de moléculas em material biológico. Energia de $10 \mathrm{eV}$ corresponde a frequiência de $2,4 \times 10^{15} \mathrm{~Hz}$, ou seja na região do ultra-violeta curto. $\mathrm{Na}$ freqüência dos fornos de microondas $\left(2,45 \times 10^{9} \mathrm{~Hz}\right)$ o fóton tem energia um milhão de vezes menor, não tendo qualquer possibilidade de provocar ionização em alimentos, animais ou pessoas expostas .

Outros termos comumente relacionados com a exposição às ondas eletromagnéticas, são: "interação", "efeito", "sensação" e "risco".

Interação é a ação do campo eletromagnético sobre o sistema biológico. É claro que, por menor que seja a energia do campo aplicado, o sistema biológico sofre uma perturbação, nem que seja em nível quântico. Efeito (em seres vivos) é uma perturbação fisiológica, mensurável ou não. Sensação (em seres humanos) é a percepção de uma perturbação fisiológica. Risco : entende-se que só há risco (ou "perigo") se for provado que o efeito possa resultar em dano imediato ou a longo prazo.

Algumas formas bem conhecidas de interação podem servir como exemplos. Sabemos que raios solares produzem efeito, com sensação, com risco. Por outro lado, os raios $X$ produzem efeito, sem sensação, com risco. A luz visível natural produz efeito, com sensação, sem risco. O campo magnético terrestre (que se saiba) não causa efeito, nem sensação, e nem apresenta risco.

Observe-se que não há unanimidade quanto aos conceitos acima expostos. Em particular, o conceito de risco, além de ter outro significado em epidemiologia, é também considerado por alguns como sendo conseqüência direta de efeito. Ou seja, detectado o, efeito, haveria risco[3][4].

Os efeitos biológicos relacionados com rádiofrequiências (RF) e microondas, podem ser de três tipos:
a) choques e queimaduras
b) efeitos atérmicos
c) efeitos térmicos.

É bem sabido que objetos podem acumular cargas induzidas por campos eletromagnéticos. Uma pessoa, ao tocar esses objetos, pode receber um choque, causado por correntes que fluem através do corpo. A intensidade da corrente depende de vários fatores que caracterizam o caminho da descarga à terra. O limiar de dor varia com as pessoas e com a frequência. Valores típicos situam-se na faixa de 50 a $100 \mathrm{~mA}$.

$O$ perigo de choques e queimaduras (sobretudo nas mãos) é maior nas RF mais baixas, devendo ser lembrado, p.ex., por operadores e encarregados da manutenção de estações de rádio-difusão. Há normas de segurança específicas para esses casos.

Classificam-se como atérmicos (ou não-térmicos) efeitos que não podem ser atribuidos a alterações da temperatura, geral ou localizada, do corpo.

A questão da existência, ou não, de efeitos atérmicos, observados em níveis de exposição muito inferiores aos fixados nas normas de segurança em vigor, tem ocupado o centro de inúmeras discussões. Na opinião da grande maioria dos pesquisadores, pode-se afirmar que : 
1) os efeitos atérmicos comprovados são reversíveis e não constituem risco. 2) muitos efeitos inicialmente classificados como atérmicos, por desconhecimento do mecanismo causador (p.ex. o efeito auditivo associado a pulsos de microondas), passaram a ser qualificados como efeitos térmicos após análise mais apurada [5].

\section{EFEITOS TÉRMICOS}

O efeito biológico mais conhecido dos campos eletromagnéticos - e mais fácil de entender, é o efeito térmico. A pele aquece ao sol. Os alimentos cozinham no forno de microondas.

Os mecanismos de interação são bem conhecidos, predominando, na faixa de RF e microondas, o fenômeno de relaxação de moléculas polares (em particular a água) sob ação do campo elétrico de alta freqüência.

Nos anos 30 e 40, desenvolveram-se geradores de RF de alta potência. Operadores desses transmissores logo observaram, talvez como fato curioso, que a exposição à RF podia produzir sensação de calor.

Da curiosidade, passou-se à preocupação. Operadores de transmissores da Marinha dos EUA queixaram-se de fraqueza, náuseas e outros efeitos, por eles atribuídos à RF. Experiências com voluntários e com animais detectaram sintomas semelhantes aos causados por febre. Todos os voluntários recuperaram-se sem dano permanente Os animais expostos geraram crias normais. Concluiu-se que os efeitos de RF sobre os seres vivos são apenas de natureza térmica e reversíveis, desde que a potência e/ou o tempo de exposição sejam limitados. Ou seja, basicamente o mesmo que ocorre com a exposição ao sol.

Nas mesmas décadas de 30 e 40 , começaram a ser determinadas, de maneira sistemática, as propriedades dielétricas dos tecidos biológicos, com o objetivo de entender os mecanismos de interação com os campos eletromagnéticos.

Algumas das primeiras aplicações industriais do aquecimento dielétrico por RF são também dessa época. Vale mencionar que no Brasil, em 1943, o Eng.Agr.M.Albuquerque Leão, do Ministério da Agricultura, publicou resultados de experiências de expurgo de grãos por meio de RF[6].

$\mathrm{Na}$ década de 40, houve um grande avanço nas aplicações de microondas, sobretudo em função do desenvolvimento do radar, utilizado na II guerra mundial.

Com o aumento considerável do número de pessoas, sobretudo militares, expostas a RF e microondas, multiplicaram-se os estudos sobre os efeitos biológicos das radiações não-ionizantes.

\section{NORMAS DE SEGURANÇA}

Em 1954 teve início um amplo programa de pesquisas (Tri-Service Program) das forças armadas americanas,com o objetivo de estabelecer normas de segurança.

Os resultados desse programa, divulgados em 1957/60, mais uma vez confirmaram a natureza térmica e reversível dos efeitos da exposição a $\mathrm{RF}$ e microondas $\mathrm{e}$ concluiram que níveis de densidade de potência acima de $100 \mathrm{~mW} / \mathrm{cm}^{2}$ (valor equivalente à exposição a sol forte na praia) são perigosos. Adotou-se então, como padrão de segurança, um nível máximo dez vezes menor, ou seja, $10 \mathrm{~mW} / \mathrm{cm}^{2}$. (Note-se que, após 2 décadas de uso sem preocupação com segurança, nenhum problema significativo de saúde - com exceção dos provocados por acidentes ocasionais, havia-se manifestado entre os operadores de radar.).

O mesmo nível de $10 \mathrm{~mW} / \mathrm{cm}^{2}$, aplicável a exposição de corpo inteiro, por tempo indeterminado, para qualquer frequiência de RF ou microondas, foi adotado em 1966, nas primeiras normas de segurança (ANSI C95.1-1966) elaboradas pelo American National Standards Institute (na época, American Standards Association).

Muitas outras normas de segurança foram elaboradas: normas nacionais, de órgãos governamentais e privados e normas internacionais.

Os critérios sobre os quais se baseiam as normas foram evoluindo com o tempo, chegando-se ao consenso na maioria da comunidade científica. Dessa forma, as divergências que porventura haviam entre as diversas normas, foram em grande parte eliminadas, pelo menos no tocante às faixas de frequiências de RF e microondas.

A principal base de avaliação dos efeitos térmicos está no fato de que os seres humanos, (assim como outros animais de sangue quente) possuem mecanismos termoreguladores, apoiados sobretudo na circulação sangüinea, que mantêm constante a temperatura do corpo. Doenças e causas externas podem provocar aumentos gerais ou localizados de temperatura. Esses aumentos, desde que não excessivos, podem ser anulados pelos mecanismos citados. Caso contrário, pode ocorrer dano, temporário ou permanente. (Experiências com animais mostram que um aumento prolongado da temperatura interna, de apenas $2{ }^{\circ} \mathrm{C}$ acima do normal, pode provocar mudanças no comportamento).

Tendo em vista a grande diversidade local de irrigação sanguínea, propriedades dielétricas e outras características do corpo humano [7], deduz-se que a "resposta" de partes do corpo a uma solicitação térmica provocada por absorção de energia eletromagnética é muito variada.

Por essa razão, qualquer critério "global", baseado em exposição de corpo inteiro, deve necessariamente incluir 
fator de segurança válido para qualquer parte do corpo. $\mathrm{Ou}$ então, devem-se estabelecer critérios específicos para as partes mais sensíveis do corpo (p. ex., para os olhos).

O conceito de razão específica de absorção (SAR) atende a essas dificuldades. A SAR é definida como sendo a derivada, no tempo, da energia (d $\mathscr{C}$ ) absorvida ou dissipada num elemento de massa $(\mathrm{dm})$, contido num elemento de volume (dV) [13], [14], [15].

$$
\begin{aligned}
& \mathrm{SAR}=\frac{\mathrm{d}}{\mathrm{dt}}\left(\frac{\mathrm{d} E}{\mathrm{dm}}\right)=\frac{\mathrm{d}}{\mathrm{dt}}\left(\frac{\mathrm{dE}}{\partial \mathrm{V}}\right) \quad \mathrm{W} / \mathrm{kg} ; \text { ou, de outra forma : } \\
& \mathrm{SAR}=\frac{\sigma}{2 \rho}\left|E_{\mathrm{i}}\right|^{2}=\frac{\mathrm{eq}_{\varepsilon} \varepsilon^{\prime \prime}}{2 \rho}\left|E_{\mathrm{i}}\right|^{2}=2,78 \times 10^{-11} \frac{\varepsilon^{\prime \prime}}{\rho} \mathrm{f}\left|E_{\mathrm{i}}\right|^{2} \quad \mathrm{~W} / \mathrm{kg}
\end{aligned}
$$

onde $E_{i}$ é o valor de pico do campo elétrico interno $(\mathrm{V} / \mathrm{m})_{3} \sigma$ a condutividade $(\mathrm{S} / \mathrm{m}), \rho$ a massa específica $\left(\mathrm{kg} / \mathrm{m}^{3}\right), \varepsilon$ " a parte imaginária da constante dielétrica complexa relativa, ou fator de perdas e $f$ a frequêencia $(\mathrm{Hz})$.

Observe-se que apesar de a SAR ser medida em W/kg, unidade de potência específica (por unidade de massa), a SAR expressa aumento, com o tempo, da energia específica, ou seja (Ws/kg)/s ou (Wh/kg)/h etc.

Por outro lado, a SAR relaciona-se diretamente com o aumento de temperatura:

$$
\frac{\mathrm{dT}}{\mathrm{dt}}=\frac{\mathrm{SAR}}{\mathrm{C}_{\mathrm{p}}}
$$

onde $\mathrm{C}_{\mathrm{p}}$ é o calor específico (J/kgK) .

Vê-se que a SAR indica a energia aplicada e o aumento de temperatura, em qualquer parte do corpo, pois $E_{\mathbf{i}}$, $\sigma$ (ou $\left.\varepsilon^{\prime \prime}\right), \rho$ e $C_{p}$, são grandezas locais. Fica assim bem caracterizada a diversidade de "respostas", já mencionada.

Observe-se que, em muitos casos - p.ex. em exposições de corpo inteiro -, emprega-se a SAR média, ou seja, o valor da potência total aplicada ao corpo exposto, dividido pela massa do mesmo. Por outro lado, emprega-se também a SAR local, ou valor da SAR num volume unitário ou massa unitária, que podem ser arbitrariamente pequenos.

$\mathrm{Na}$ determinação da SAR empregam-se métodos experimentais ou métodos numéricos de simulação.

A medida direta da SAR em seres humanos é em geral difícil ou impossível, apesar de haver registro de experiências limitadas com voluntários.

No caso de pequenos animais, a SAR média, de corpo inteiro, pode ser medida comparando-se a potência de RF absorvida num volume fechado, com ou sem a presença do animal.

O desenvolvimento de sensores de temperatura que não interferem com o campo elétrico (termistores com fios de ligação de resistência elevada, sensores com fibras ópticas etc.), permite, em muitos casos, a medida direta da SAR local, em pequenos volumes no interior do corpo, através do acréscimo de temperatura .

Muita informação sobre a SAR é obtida por medidas realizadas em modelos, em escala natural ou reduzida, construídos com material transparente a RF, com a forma do animal, ou do homem, ou de partes do corpo em estudo e preenchidos com material inerte tendo as mesmas propriedades dielétricas e térmicas do material biológico.

Inúmeras misturas de materiais inorgânicos foram formuladas para simular ossos, músculos, gordura e outros tecidos biológicos [13] .

Com a generalização do uso de computadores e em particular desde 1990, foram utilizados métodos computacionais poderosos (método dos momentos, método de diferenças finitas no domínio do tempo FDTD etc.), cuja plena aplicação tornou-se possível com o advento de processadores de grande capacidade e velocidade.

Com esses recursos, valores da SAR podem ser calculados por meio de modelos subdivididos em grande número de células, com dimensões tão pequenas $\mathrm{e}$ variações de propriedades tão amplas, quanto a capacidade de cálculo o permitir.

\section{ALGUNS RESULTADOS}

Apresentamos alguns resultados típicos das medidas diretas e das simulações, freqüentemente reproduzidos em publicaçōes que tratam de efeitos biológicos de campos eletromagnéticos.

A fig. 2 mostra a variação da SAR média, em função da freqüência, para três espécies animais, expostas a uma onda plana uniforme, com polarização $\mathrm{E}$ (ou seja, campo elétrico paralelo ao eixo maior do corpo) e densidade de potência de $1 \mathrm{~mW} / \mathrm{cm}^{2}$.[14]

Observa-se que há claramente uma freqüência de máxima absorção, ou de ressonância, em cada caso. Para o homem, essa freqüência situa-se próxima a $70 \sim 80 \mathrm{MHz}$, enquanto para um camundongo o máximo de absorção ocorre em $2450 \mathrm{MHz}$.

Nessa mesma freqüência (por sinal, a freqüência utilizada nos fornos de microondas), a SAR média, para o homem, é cerca de cem vezes menor do que para o camundongo exposto à mesma onda plana. Depreendese daí, p.ex., que um aumento de temperatura observado num camundongo exposto a uma dada densidade de potência em $2450 \mathrm{MHz}$, só pode ocorrer num homem sujeito a níveis de potência muito mais altos (Este fato, aparentemente óbvio, nem sempre foi considerado no passado, antes da introdução sistemática do conceito de $\mathrm{SAR}$, o que levou a erros, ao se querer estender, a seres humanos, resultados experimentais baseados em valores 


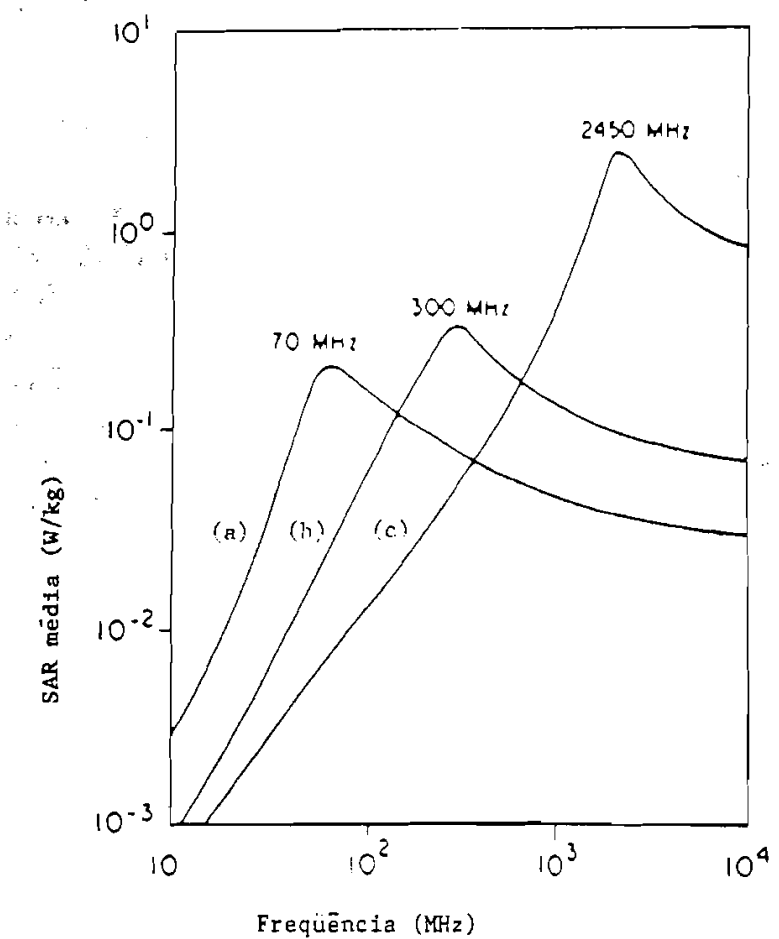

fig.2 - SAR média para exposição a onda plana uniforme com polarização $E$ e densidade de potência de $1 \mathrm{~mW} / \mathrm{cm}^{2}$.

\section{(a ) homem; (b ) macaco ; (c) camundongo}

de densidade de potência ou de campos externos aplicados a animais de pequeno porte).

Grande número de experiências com animais foram realizadas para caracterizar os limiares de risco da SAR média para produzir determinados efeitos biológicos.

No estado atual do conhecimento, pode-se admitir que valores de SAR média entre 1 e $4 \mathrm{~W} / \mathrm{kg}$ produzem efeitos biológicos mensuráveis, aceitando-se como 4 $\mathrm{W} / \mathrm{kg}$ o valor mínimo da SAR média para que haja risco de saúde para o homem. Observe-se que esse valor eqüivale a 2,5 ou 3 vezes a dissipação normal de calor pelo corpo, em repouso .

O limiar de $4 \mathrm{~W} / \mathrm{kg}$ serve de base para muitas normas recentes de segurança.

Em particular, as normas ACGIH (só ocupacionais 1993), ANSI/IEEE (1992), CENELEC (1995) e IRPA (1993)[8]a[12], incorporam fatores de segurança conservadores, que limitam a SAR média, isto é a exposição de corpo inteiro, a $0,4 \mathrm{~W} / \mathrm{kg}$ em condições controladas ( quando a pessoa está ciente de que está exposta, o que ocorre tipicamente com operadores de equipamentos) e a $0,08 \mathrm{~W} / \mathrm{kg}$ em condições não controladas ( o que vale para "a população em geral").

Como valores típicos, na faixa de frequiências $f$ entre 300 e $3000 \mathrm{MHz}$, em termos de densidade de potência irradiando o corpo inteiro, a norma ANSI [9] estipula um máximo de $\mathrm{f} / 300 \mathrm{~mW} / \mathrm{cm}^{2}$ em condições controladas e $\mathrm{f} / 1500 \mathrm{~mW} / \mathrm{cm}^{2}$ em condições não controladas. Por exemplo, na frequiência hoje em dia "familiar" de $2450 \mathrm{MHz}$, esses máximos correspondem a 8 e 1,6 $\mathrm{mW} / \mathrm{cm}^{2}$, respectivamente (v.fig.3)

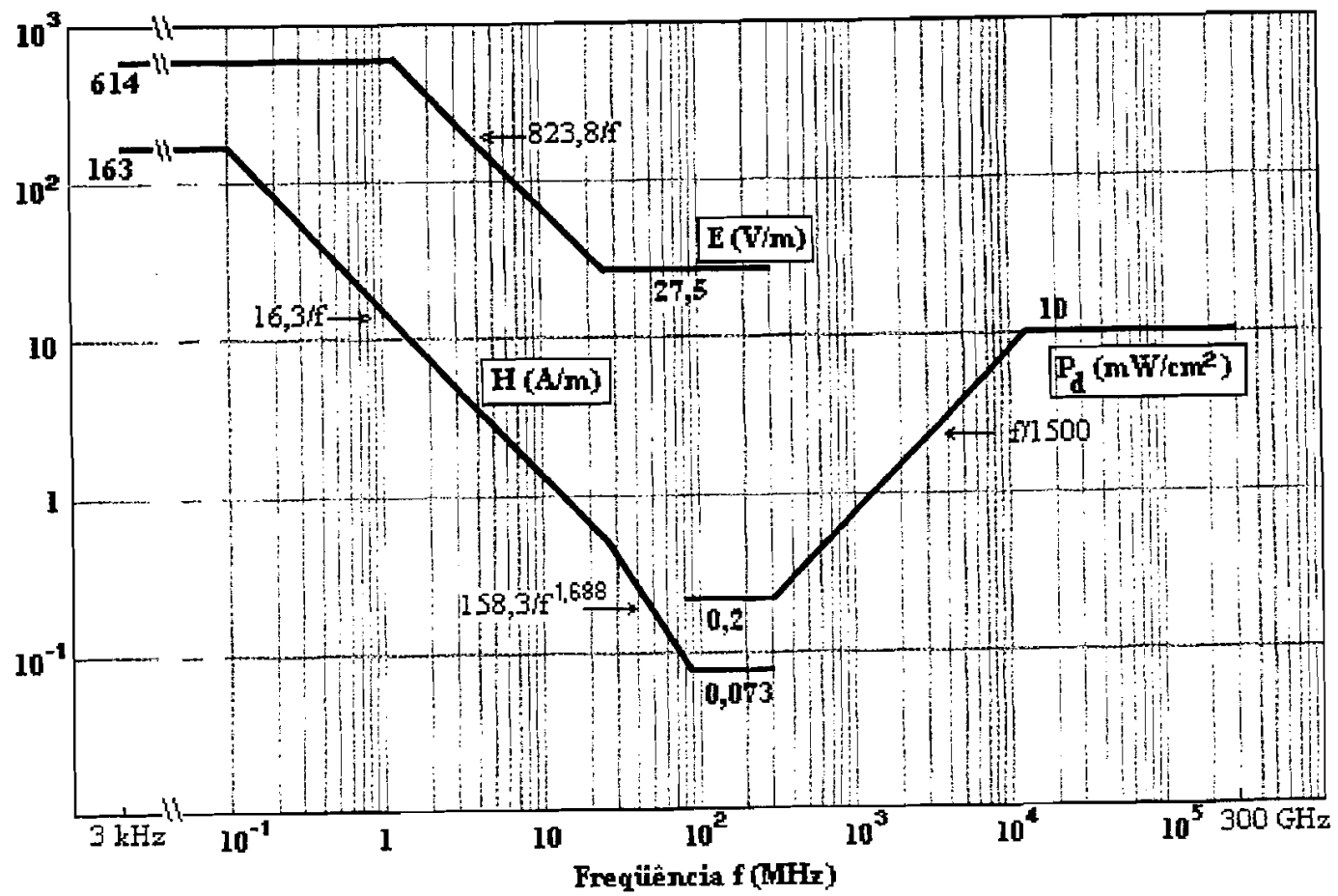

Fig 3 - Niveis máximos de exposição - ambiente não controlado (IEEE/ANSI) 


\section{TELEFONIA SEM FIO}

O grande desenvolvimento da telefonia celular e de outras formas de telecomunicações pessoais sem fio, trouxe novas preocupações com relação à proteção contra possíveis riscos associados à exposição dos usuários aos campos eletromagnéticos [16].

De fato, com raras exceções, em todos os estudos realizados até pouco anos atrás, supôs-se que o objeto exposto recebia energia eletromagnética na forma de uma onda plana, ou seja, supôs-se que o objeto encontrava-se na zona de campo remoto da antena emissora.

Na telefonia celular, a situaçāo é bem diversa. A antena e o próprio transceptor, encontram-se muito próximos da cabeça. Além da configuração dos campos nada ter a ver com uma onda plana uniforme, uma fração não desprezível da energia irradiada pela antena é absorvida nas mãos, na cabeça, incluindo os olhos e no pescoço.

Nas normas de segurança existentes, essa situação não é prevista.

Resultados de grande número de medidas e simulações matemáticas tem sido publicados recentemente. Vários grupos de pesquisadores estão utilizando métodos numéricos para avaliar a influência, na SAR local em várias partes expostas do corpo, de vários aspectos operacionais da telefonia celular, entre os quais a forma do transceptor, o tipo e a localização da antena [17]., [18]

De maneira geral, os trabalhos publicados indicam que 10 a $50 \%$ da potência irradiada pelo transmissor podem ser absorvidos pela cabeça, com valor máximo de 1 a 8 $\mathrm{W} / \mathrm{kg}$ por $1 \mathrm{~W}$ de potência, dependendo da distância entre cabeça e antena (variando de $1 \mathrm{a} 4 \mathrm{~cm}$ ).

Antenas de nova concepção, integradas ao corpo do transceptor, foram desenvolvidas e - em igualdade de potência, produzem SAR inferior a que ocorre com os monopolos usuais.

Com a expectativa da futura utilização de novos sistemas de transmissão e de novas faixas de frequiências, já estão em curso simulações que avaliam o impacto dessas novas condições, de forma a garantir que os novos equipamentos poderão ser operados com segurança pelos futuros usuários.

Pelo que resulta da maioria dos trabalhos confiáveis publicados, pode-se concluir que não há sérias preocupações com o uso de telefones celulares [5].

Poderão ocorrer desenvolvimentos futuros, não só de equipamentos, como de técnicas de modulação digital, que necessitarão novos estudos para garantir o uso seguro dos telefones, mas tudo leva a crer que, como ocorreu com todas as inovações tecnológicas deste século, após um periodo de dúvidas, a aceitação consciente e geral ocorrerá também para os telefones celulares.

\section{CONCLUSÃO}

A história mostra que a introdução de uma nova tecnologia pode gerar reações diversas, que vão do entusiasmo à descrença, da adesão à oposição [19], [20].Oposição muitas vezes desinteressada, mas que pode também ser cega e até mesmo tendenciosa. Este último, é o caso dos que exploram as dúvidas e os temores dos desinformados, para se autopromoverem e até mesmo para auferirem lucro (p.ex., com a publicação de livros de cunho "anti-tecnológico" sensacionalista).

Não é de admirar que leigos tenham dúvidas com relação aos efeitos biológicos dos campos eletromagnéticos, já que há dúvidas na própria comunidade científica, o que é natural e faz parte da atividade de pesquisa. Trabalhos científicos, frequientemente contraditórios, são publicados (às vezes às pressas) e submetidos à crítica dos pares. Muitos trabalhos caem no esquecimento, por falta de reprodutibilidade, ou por apresentarem falhas conceituais ou metodológicas. Os que restam, formam o corpo de conhecimentos aceito pela maioria (mas não necessariamente pela totalidade) dos pesquisadores de determinada área.

As pesquisas sobre efeitos biológicos não fogem à regra. Existe uma agravante. Assim que é publicado algum trabalho que aponta para possíveis riscos associados à exposição a campos eletromagnéticos, esse trabalho merece ampla divulgação, inclusive por órgãos de comunicação de massa. Vice-versa, quando o mesmo trabalho é contestado, suas falhas evidenciadas e suas conclusões consideradas errôneas, dificilmente os órgãos de comunicação se interessam em divulgar esse fato, que permanece restrito ao âmbito das publicações científicas.

Neste trabalho, procurou-se fazer um apanhado das bases históricas e dos critérios atuais sobre os quais se apoiam as normas de segurança mais recentes (até julho de 1996) para a exposição a RF e microondas.

Em particular, enfatizou-se a importância do conceito de SAR - razão específica de absorção e dos métodos experimentais e computacionais que permitem a sua avaliação.

Como tópico relacionado e de grande atualidade, procurou-se reportar o estágio atual dos trabalhos sobre a segurança dos usuários de telefones celulares.

Tudo o que foi aqui relatado é apenas uma pequena parte do que pode ser encontrado na vasta literatura sobre o assunto, facilmente acessível a todos os que se interessam por eletromagnetismo, saúde e tópicos correlatos.

Espera-se que este resumo sirva de estímulo para a busca mais detalhada de conhecimentos sobre o tema. 


\section{AGRADECIMENTOS}

$O$ autor agradece o apoio recebido do IMT e da FAPESP, ao longo de mais de vinte anos de atividades do Laboratório de Microondas do IMT. Especial reconhecimento é também devido aos Profs. Maria e Stanislaw Stuchly, da Universidade de Victoria, B.C.,Canada, pelas muitas e valiosas informações fornecidas em contatos pessoais e em correspondências.

\section{NOTAS E REFERÊNCIAS}

[1] - Muita informação histórica pode ser encontrada em Bren,S.P.A."Historical Introduction to EMF Health Effects" - IEEE Engineering in Medicine and Biology Magazine, vol.15 N. 4, 1996 - (O mesmo número da revista contem vários artigos sobre efeitos biológicos de campos eletromagnéticos, com extensa bibliografia).

[2] - Reproduzido em Scientific American, julho 1996

[3] As normas em vigor na ex-União Soviética e nos países do leste europeu baseiam-se em conceitos de efeito e risco mais restritivos do que os empregados no ocidente, com ênfase em possíveis alterações comportamentais que não são consideradas de risco por pesquisadores ocidentais.

[4] - Um tópico que foge ao escopo deste trabalho, mas que cabe mencionar, é o da interpretação de normas de emissão de equipamento versus normas de exposição de pessoas. P.ex., as normas aplicáveis a equipamentos limitam a $5 \mathrm{~mW} / \mathrm{cm}^{2}$, durante toda a vida útil $(1 \mathrm{~mW} / \mathrm{cm} 2$ ao sair da fábrica) a emissão de um forno de microondas, medida a $5 \mathrm{~cm}$ de distância de qualquer ponto do aparelho. $\hat{E}$ claro que isso não significa que o usuário de um forno fique exposto aos mesmos $5 \mathrm{~mW} / \mathrm{cm}^{2}$, visto que a energia eletromagnética decai com o quadrado da distância e ninguém habitualmente mantém uma distância de apenas 5 $\mathrm{cm}$ de um forno em funcionamento.

[5] - Um dos poucos efeitos verificados in vitro e geralmente aceitos como atérmicos é a alteração do fluxo de ions de cálcio através de membranas celulares, sob a ação de campos de RF (140 ou 450 $\mathrm{MHz}$ ) modulados com baixa frequiência. Esse efeito ocorre somente para determinados níveis de potência $\left(0,1\right.$ a $\left.1 \mathrm{~mW} / \mathrm{cm}^{2}\right)$ e de frequiência de modulação (6 a $20 \mathrm{~Hz}$ ). Apesar de não haver evidência de que esse efeito possa apresentar risco, ou seja irreversível, continuam as investigações sobre o fenômeno, tendo em vista a possível exposição de pessoas a campos de RF e microondas modulados por baixas freqüências, como ocorre nos telefones celulares digitais.

[6] - Leão, M.A. " O expurgo de grãos pelas ondas curtas." Boletim do Ministério da Agricultura, Rio de Janeiro, maio 1943.

[7] - A tabela I mostra alguns valores médios encontrados na literatura, válidos para frequiências próximas de $900 \mathrm{MHz}$ (telefonia celular) e usados em simulações.

[8] - ACGIH "Annual Report of the Committees on Threshold Limit Values and Biological Exposure Indices." American Conference of Governmental Industrial Hygienists, Cincinnati, 1993.

[9] - ANSU/IEEE C95.1 "IEEE Standard for Safety Levels with Respect to Human Exposure to RF Electromagnetic Fields, $3 \mathrm{kHz}$ to $300 \mathrm{GHz}$." Institute of Electrical and Electronic Engineers, New York, 1992.

[10]- CENELEC ENV 50166-2 "Human Exposure to Electromagnetic Fields. High Frequency $(10 \mathrm{kHz}$ to $300 \mathrm{GHz}$ ). European Committee for Electrotechnical Standardization, Brussels, 1995.

[11] - EUB Tech 3278-E "Radiofrequency Radiation Hazards - Exposure limits and their implications for broadcasters". European Broadcasting Union, Geneva, 1995 (Estudo comparativo das principais normas).

[12] - IRPA "Electromagnetic Fields (300 Hz to 300 $\mathrm{GHz}$, Environmental Health Criteria, 137." International Radiation Protection Association, WHO, Geneve, 1993.

[13] - Durney,C.H., Massoudi,H. and Iskander,M.F. Radiofrequency Radiation Dosimetry Handbook USAF School of Medicine Report TR-85-73, 1986

[14] - Stuchly, M.A. "Health Effects of Exposure to Electromagnetic Fields." Proceedings, 1995 IEEE Aerospace Applications Conference, pp.351-368, Feb.1995.

[15] - Greene,M.W. (ed.) - Non-Ionizing Radiation -

Tabela I

\begin{tabular}{|l|l|l|l|l|l|l|}
\hline Tecido & Músculo & Gordura & Pele & Sangue & Córnea & Cristalino \\
\hline Const.dielétrica relativa $\varepsilon_{\mathrm{r}}$ & 51,0 & 7,2 & 35,0 & 64,0 & 52,0 & 45,0 \\
\hline Condutividade $\sigma(\mathrm{S} / \mathrm{m})$ & 1,35 & 0,16 & 0,60 & 1,25 & 1,80 & 0,75 \\
\hline Massa específica $\rho\left(\mathrm{g} / \mathrm{cm}^{3}\right)$ & 1,04 & 1,10 & 1,10 & 1,06 & 1,02 & $1,0 \Sigma$ \\
\hline
\end{tabular}


Proceedings, 2nd International Non-Ionizing Workshop, Vancouver,B.C.,1992.

[16]- Stuchly, M.A. "Mobile Communication Systems and Biological Effects on their Users." , URSI Radio Science Bulletin No.275, pp 7-13, Dec.1995.

[17] - Jensen,M.A. and Rahmat-Samii,Y. "EM Interaction of Handset Antennas and a Human in Personal Communications." Proc.IEEE, vol.83, pp.7-17, 1995

[18] - Gandhi,O.P. and Chen,J.Y. "Electromagnetic Absorption in the Human Head from Experimental 6-GHz Handheld Transceivers." IEEE Trans.Electromagnetic Compatibility vol.37, pp.547-558, Nov.1995

[19]- Fischetti,M. "The cellular phone scare." IEEE Spectrum, pp.43-47, June 1993

[20]- Jauchen,J.R. "Alleged Health Effects of Electromagnetic Fields: the Misconceptions Continue."Journal of Microwave Power and Electromagnetic Energy, vol.30, pp.165-177, 1995.

\section{DADOS BIOGRÁFICOS}

José Thomaz Senise é Professor da Escola de Engenharia Mauá do Instituto Mauá de Tecnologia e Chefe da Divisão Técnica de Telecomunicações e Microondas do Centro de Pesquisas do IMT. Engenheiro (Univ.de S.Paulo, 1947) e Ph.D. em Engenharia Elétrica (Stanford University, 1959). Recebeu bolsa do British Industries Council para treinamento industrial em Manchester, Inglaterra (19481950). Recebeu bolsa do Conselho Nacional de Pesquisas para pós-graduação na Stanford University (1954-1957). Research Associate do Microwave Laboratory, Stanford University (1958). Professor do Instituto Tecnológico de Aeronáutica (1951 a 1966). Professor Colaborador (MS-6) da Escola Politécnica da USP (1968 a 1978). Professor Visitante da University of Ottawa, Canada (1986). Professor da Escola de Engenharia Mauá, desde 1966. A partir de 1970, com recursos provenientes da Fundação de Amparo à Pesquisa do Estado de São Paulo, de empresas e do próprio IMT, dedica-se a atividades de pesquisa, desenvolvimento e formação de pessoal, na área de aplicações industriais de microondas, tendo registrado quatro patentes. Representante Nacional na Comissão K - Eletromagnetismo em Biologia e Medicina, da URSI. Editor Associado do Journal of Microwave Power and Electromagnetic Energy, do International Microwave Power Institute. Sócio fundador e primeiro Presidente da SBMO-Sociedade Brasileira de Microondas e Optoeletrônica, da qual é Presidente de Honra. Life Member do IEEE.

Endereço:

Instituto Mauá de Tecnologia

09580-900 S.Caetano do Sul, SP

Tel: (011) 741-3000 Fax (011)741-3131

e-mail: maua@eu.ansp.br 